\title{
The herbal medicine rikkunshito exhibits strong and differential adsorption properties for bile salts
}

\author{
YOSHIO ARAKI ${ }^{1}$, KEN-ICHI MUKAISHO $^{1}$, YOSHIHIDE FUJIYAMA ${ }^{2}$, \\ TAKANORI HATTORI $^{1}$ and HIROYUKI SUGIHARA ${ }^{1}$ \\ Departments of ${ }^{1}$ Pathology, and ${ }^{2}$ Internal Medicine, Shiga University of Medical Science, \\ Seta Tsukinowa-cho, Otsu, Shiga 520-2192, Japan
}

Received October 31, 2011; Accepted December 1, 2011

DOI: $10.3892 /$ etm.2012.478

\begin{abstract}
Anti-secretory drugs, particularly proton pump inhibitors (PPIs), are the preferred treatment agents for patients with gastroesophageal reflux disease (GERD). However, refractory GERD, which may manifest as an incomplete or lack of response to PPI therapy, is common. Despite the administration of PPIs for symptomatic control, duodenogastroesophageal reflux (DGER) containing bile is successfully controlled in only one-third of patients. It has previously been reported that the traditional Japanese herbal medicine rikkunshito, which has a prokinetic action on gastric emptying, exhibits clinically therapeutic effects against GERD and DGER that does not respond to PPIs. However, the precise mechanisms responsible for the effects of rikkunshito are still unknown. It has been suggested that the cytotoxicity of the bile salts in the gut lumen is important in GERD and DGER. The aim of the present study was to investigate whether rikkunshito is able to adsorb bile salts through the mechanism by which it ameliorates the symptoms of GERD and DGER. The binding capacities of rikkunshito for bile salts were measured using Langmuir's method. The morphology of rikkunshito was also observed by light microscopy. Rikkunshito strongly adsorbed bile salts. The binding capabilities of rikkunshito were far beyond those of a typical dietary fiber, $\alpha$-cellulose, or an oral adsorbent. In addition, rikkunshito had higher binding capacities for hydrophobic bile salts as compared with hydrophilic bile salts. In conclusion, rikkunshito has a great capacity to adsorb bile salts. This may be part of the mechanism(s) responsible for the therapeutic effects of rikkunshito in patients with GERD and DGER.
\end{abstract}

Correspondence to: Dr Yoshio Araki, Department of Pathology, Shiga University of Medical Science, Seta Tsukinowa-cho, Otsu, Shiga 520-2192, Japan

E-mail: yir0828@belle.shiga-med.ac.jp

Key words: rikkunshito, herbal medicine, dietary fiber, bile salt, adsorption

\section{Introduction}

In recent decades, gastroesophageal reflux disease (GERD) has become a common disorder in the USA and Western Europe (1). Anti-secretory therapies, especially proton pump inhibitors (PPIs), are the preferred treatment for patients with GERD. However, it has been estimated that between 10 and $40 \%$ of patients with GERD fail, either partially or completely, to respond symptomatically to standard doses of PPIs (2-5). The majority of bile reflux occurs concomitantly with acid reflux events and it is believed that the acid rather than the bile is the dominant factor responsible for the symptoms of GERD $(6,7)$. However, certain studies have suggested that persistent typical and atypical GERD symptoms refractory to PPIs might be due to less acidic or non-acidic reflux (8). In addition, experimental data support a role for persistent bile acids in the refluxate as factors potentially involved in refractory heartburn. Although PPI therapy reduces the occurrence of acid as well as bile reflux (9), it has been shown that complete acid suppression does not guarantee the elimination of duodenogastroesophageal reflux (DGER) (10). Taken together, it is possible that bile reflux accounts for at least some of the non-acid reflux symptoms (11).

Previously, there were no data available concerning the value of administering a promotility drug to patients who have failed PPI therapy. However, in patients receiving PPI therapy who have delayed gastric emptying and persistent GERD symptoms, the use of a promotility drug is an attractive option. Over the years, the traditional Japanese herbal medicine rikkunshito, which is used to treat various disorders of the gastrointestinal tract, including functional dyspepsia, gastroesophageal reflux, dyspeptic symptoms of post-gastrointestinal surgery and chemotherapy-induced nausea, has been used to treat the symptoms of GERD and DGER and studies concerning its efficacy have been published (12-15). Rikkunshito has a prokinetic action on gastric emptying and its pharmacological action is closely correlated with an increase in plasma-active ghrelin levels, which stimulates gastric motility (16). However, it is unknown whether rikkunshito exerts its effects against GERD and DGER via prokinetic actions alone.

Rikkunshito is a type of dietary fiber derived from medicinal plants. In general, medicinal plants mainly consist of carbohydrates, insulin, fats, proteins, wax, mucus, gum resin, 
balsam resin, essential oils, triterpenes, saponins, tannins, lignin, lignans, glycosides, alkaloids and calcium salts. In recent decades, there has been an increased interest in dietary fiber due to its apparently beneficial effects on the human gastrointestinal tract, which include improving constipation, reducing serum cholesterol levels and excreting carcinogenic compounds into the feces $(17,18)$. It is widely accepted that the beneficial effects of dietary fiber are mainly due to its binding or bulking characteristics (19). Dietary fiber refers to plant cell wall components and consists mainly of two types of fiber: soluble fiber (pectin, $\beta$-D-glucans, fructans, oligosaccharides, certain hemicelluloses, guar and gums) and insoluble fiber (hemicellulose, cellulose and lignin) (20). Dietary fibers cannot be digested by human or other mammalian digestive enzymes and can only be degraded by anaerobic bacteria located in the large intestine. We have previously focused on a certain type of dietary fiber, germinated barley foodstuff (GBF), as a therapeutic agent for inflammatory bowel disease (21-24). In the process of investigating the therapeutic mechanisms of $\mathrm{GBF}$, we discovered that one of its major properties was its capacity to adsorb bile salts (25), thus eliminating bile salts from the gut lumen and ameliorating colitis.

Therefore, the aim of the present study was to investigate whether rikkunshito is able to adsorb bile salts in vitro and, if so, to establish whether this capability contributes to its therapeutic effects in reducing the symptoms of patients with GERD and DGER.

\section{Materials and methods}

Chemicals. Dicyclohexano-18-crown-6, 4-bromomethyl6,7-dimethoxycoumarin, cholate (CA), taurocholate (T-CA), deoxycholate (DCA), taurodeoxycholate (T-DCA) and $\alpha$-cellulose were purchased from Nacalai Tesque, Inc. (Kyoto, Japan). All these bile salts were of analytical grade.

Rikkunshito. Rikkunshito was used in the form of a powdered mixture of eight types of crude herbs, sojutsu (Atractylodis lanceae rhizoma), ninjin (Ginseng radix), hange (Pinelliae tuber), bukuryo (Hoelen), taiso (Zizyphi fructus), chinpi (Aurantii nobilis pericarpium), kanzo (Glycyrrhizae radix) and shokyo (Zingiberis rhizoma). Rikkunshito was supplied by Tsumura and Co. (Tokyo, Japan).

Microscopic observations of rikkunshito. We observed the morphology of the two dietary fibers, rikkunshito and $\alpha$-cellulose, microscopically. The morphological differences between the dry and the wet forms are important. Therefore, when observing the dry form, we inspected the fibers directly. In the observation of the wet form, $50 \mathrm{mg}$ of each dietary fiber was immersed in $10 \mathrm{ml}$ of distilled water for $5 \mathrm{~min}$ and was then set under a cover glass for direct inspection. We used the light microscope Olympus BX 50 (Olympus Optical Co., Ltd., Tokyo, Japan) to carry out these observations.

Binding capacities of rikkunshito for bile salts. We used bile salt solutions of concentrations ranging from $100 \mu \mathrm{M}$ to $1 \mathrm{mM}$ in the binding experiment. In addition, $\alpha$-cellulose was used in order to compare the binding capacity of rikkunshito with other types of fiber. The binding experiment was carried out according to our previous method (25). Briefly, $50 \mathrm{mg}$ of rikkunshito or $\alpha$-cellulose was placed into a glass-stoppered conical flask with $5 \mathrm{ml}$ of water. The flasks were then closed securely, mechanically agitated at $25^{\circ} \mathrm{C}$ for $30 \mathrm{~min}$ and the supernatant subjected to filtration $(0.45-\mu \mathrm{m}$ pore size). The bile salts in the supernatants were analyzed as their fluorescent dimethoxy coumarin esters using high-performance liquid chromatography (HPLC) according to our previous method. Briefly, following the drying of the supernatants using a vacuum pump, a $100 \mu \mathrm{l}$ aliquot of acetonitrile was added, followed by $40 \mu \mathrm{l}$ of dicyclohexano-18-crown-6 $(1.5 \mathrm{mg} / \mathrm{ml}$ acetonitrile) and $40 \mu \mathrm{l}$ of 4-bromomethyl-6,7-dimethoxycoumarin $(3.0 \mathrm{mg} / \mathrm{ml})$. The tubes were then sealed with parafilm and placed in a heated water bath at $60^{\circ} \mathrm{C}$ for $30 \mathrm{~min}$. Next, the solution was centrifuged at $14,000 \mathrm{rpm}$ for $10 \mathrm{~min}$ and $5 \mu \mathrm{l}$ of the supernatant was injected into the HPLC column. We used a reverse-phase column (Cosmosil 5C18-MS, 4.6 mm IDx15 cm long, Nacalai Tesque Inc.) and two solvents as the mobile phase. Solvent A was a mixture of water, acetonitrile and methanol at a ratio of 3:2:1. Solvent B was a mixture of acetonitrile and methanol at a ratio of 2:1. A $1 \mathrm{ml}$ aliquot of $7.6 \mathrm{M}$ ammonium acetate was added to each $500 \mathrm{ml}$ of solvents $\mathrm{A}$ and $\mathrm{B}$. The flow rate was constant at $0.6 \mathrm{ml} / \mathrm{min}$. The gradient elution program began with $100 \%$ solvent A and the proportion of solvent B was gradually increased from 0 to $95 \%$ over a 45 -min period, then kept constant for an additional 10 min prior to recycling to the initial conditions. A fluorescence detector RF-535 (Shimadzu, Kyoto, Japan) was set at an excitation wavelength of $340 \mathrm{~nm}$ and an emission wavelength of $430 \mathrm{~nm}$. The bile salt peaks were quantified by comparing the areas to standard curves produced by chromatographing known quantities of bile salt standards under similar conditions.

Following the measurement of the bile salt concentrations, we obtained adsorption isotherms for the binding of individual bile salts to rikkunshito, according to a previous method using a Langmuir-type equation (26,27):

$$
\begin{aligned}
& \text { Equation 1: } \mathrm{x} / \mathrm{m}=\mathrm{k}_{1} \mathrm{k}_{2} /\left(1+\mathrm{k}_{1} \mathrm{Ceq}\right) \\
& \text { Equation 2: } \mathrm{Ceq} /(\mathrm{x} / \mathrm{m})=1 / \mathrm{k}_{1} \mathrm{k}_{2}+\mathrm{Ceq} / \mathrm{k}_{2}
\end{aligned}
$$

where Ceq is the concentration of the bile salt remaining in solution at equilibrium, $\mathrm{x}$ is the amount of bile salt bound to the rikkunshito and $\mathrm{m}$ is the amount of rikkunshito used. A plot of $\mathrm{Ceq} /(\mathrm{x} / \mathrm{m})$ versus Ceq should yield a straight line, from which we may obtain the constants $\mathrm{k}_{1}$ (the adsorption coefficient) and $\mathrm{k}_{2}$ (the maximum binding capacity).

\section{Results}

Macroscopic and microscopic observations of rikkunshito. Fig. 1A shows the macroscopic appearance of rikkunshito, which is a yellow and fairly rough powder. Fig. 1B shows the macroscopic appearance of $\alpha$-cellulose, which is a white and smooth powder. Fig. 2A shows the microscopic appearance of rikkunshito. Rikkunshito has an amorphous structure of spherical particles $\sim 40-80 \mu \mathrm{m}$ in diameter. Fig. $2 \mathrm{~B}$ shows the microscopic appearance of $\alpha$-cellulose, which has an amorphous structure of long, rod-shaped particles of $\sim 20 \mu \mathrm{m}$ by $40-240 \mu \mathrm{m}$. Notably, if the rikkunshito was immersed in 


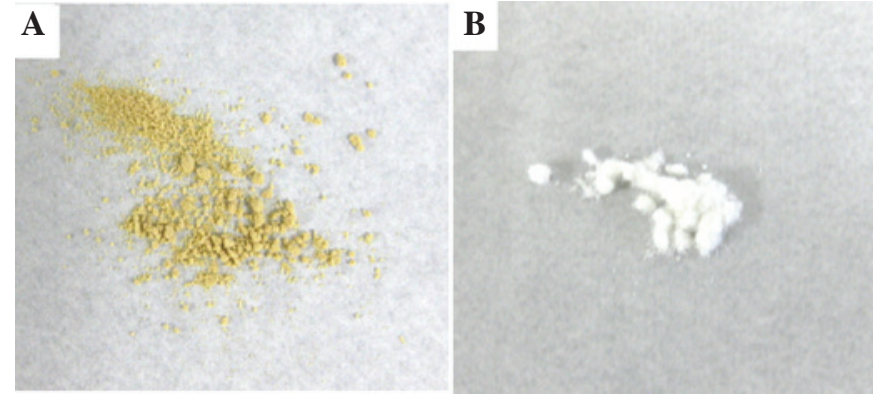

Figure 1. Macroscopic observation of rikkunshito and $\alpha$-cellulose. (A) Macroscopic observation of rikkunshito. Rikkunshito is a yellow and rough powder. (B) Macroscopic observation of $\alpha$-cellulose. $\alpha$-cellulose is a white and fairly smooth powder.

A

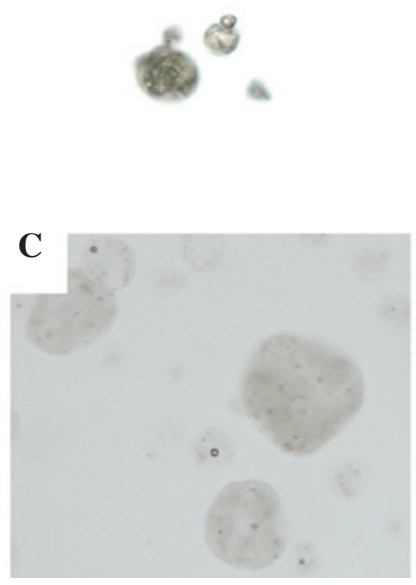

B
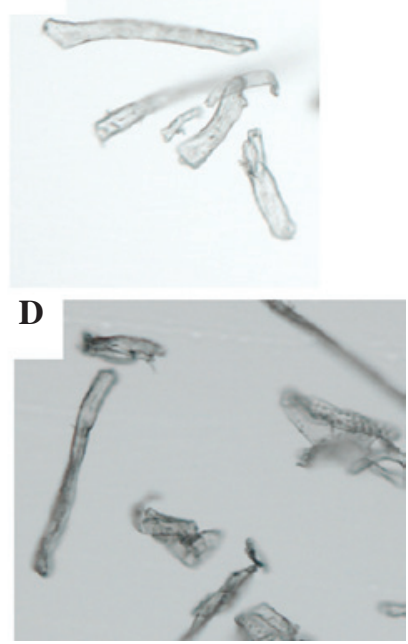

Figure 2. Microscopic observation of rikkunshito and $\alpha$-cellulose (x100 magnification). (A) Microscopic observation of rikkunshito. Rikkunshito has an amorphous structure of spherical particles of $\sim 40-80 \mu \mathrm{m}$ in diameter. (B) Microscopic observation of $\alpha$-cellulose. $\alpha$-cellulose has an amorphous structure of long rod-shaped particles of $\sim 20 \mu \mathrm{m}$ by $40-240 \mu \mathrm{m}$. (C) When rikkunshito was immersed in distilled water, the particles swelled and increased $\sim 3$-fold in diameter. (D) $\alpha$-cellulose did not swell when immersed in distilled water.

distilled water, the particles swelled rapidly and their diameter increased $\sim 3$-fold (Fig. 2C). However, $\alpha$-cellulose did not swell in distilled water to any appreciable extent (Fig. 2D).

Binding capacities of rikkunshito for bile salts. Figs. 3 and 4 show the adsorption isotherms of rikkunshito for individual conjugated and unconjugated bile salts according to equations 1 and 2, respectively. The curves had a tendency to reach a plateau at high Ceq values. The adsorption constants $\mathrm{k}_{1}$ and $\mathrm{k}_{2}$, obtained from the intercept and slope values of Figs. 3 and 4, are listed in Table I. Rikkunshito adsorbed individual conjugated and unconjugated bile salts strongly compared with $\alpha$-cellulose. In particular, unconjugated CA and DCA were more strongly adsorbed by rikkunshito than their conjugated counterparts, T-CA and T-DCA. CA and DCA had maximum binding capacities of $748.0 \times 10^{-6}$ and $752.0 \times 10^{-6} \mathrm{~mol} / \mathrm{g}$, respectively. However, T-CA and T-DCA

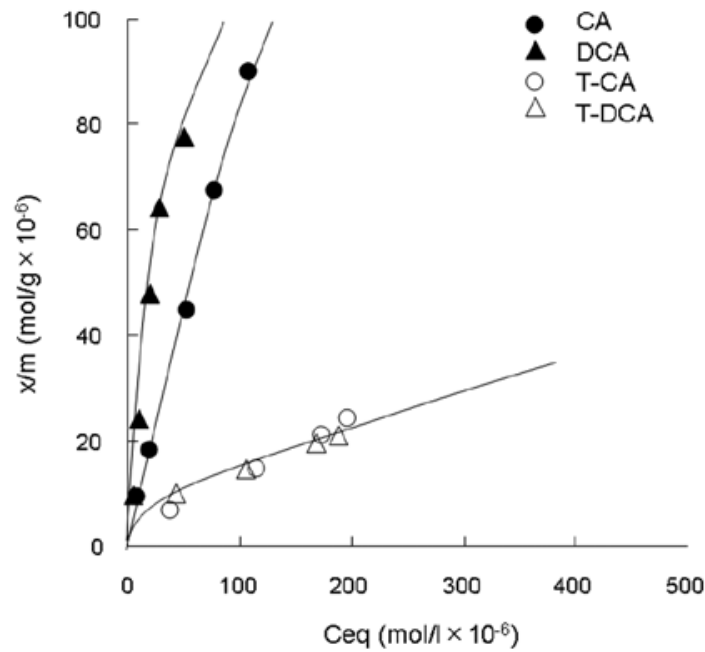

Figure 3. Langmuir adsorption isotherms for the binding of bile salts. The Langmuir adsorption isotherms for the binding of unconjugated or conjugated bile salts to rikkunshito at $25^{\circ} \mathrm{C}$ are shown. The isotherms were calculated using equation 1 (described in Materials and methods). CA, cholate; DCA, deoxycholate; T-CA, taurocholate; T-DCA, taurodeoxycholate.

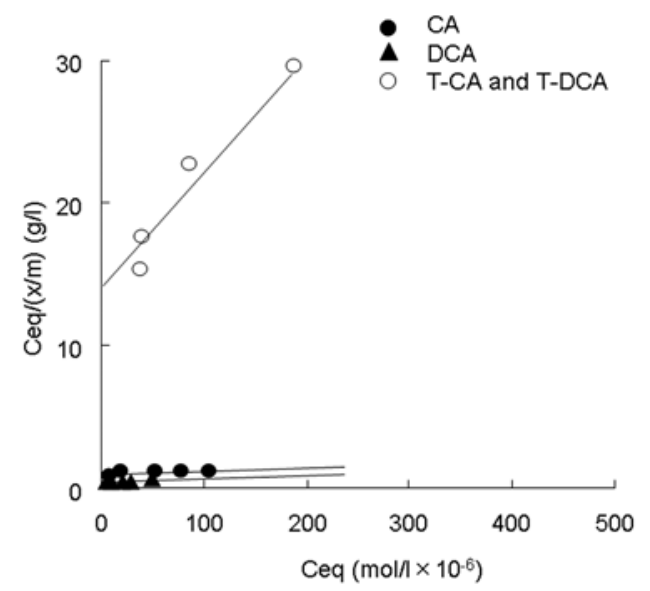

Figure 4. Langmuir adsorption isotherms for the binding of bile salts. The Langmuir adsorption isotherms for the binding of unconjugated or conjugated bile salts to rikkunshito at $25^{\circ} \mathrm{C}$ are shown. The isotherms were calculated using equation 2 (described in Materials and methods). CA, cholate; DCA, deoxycholate; T-CA, taurocholate; T-DCA, taurodeoxycholate.

Table I. Langmuir adsorption constants for the binding of bile salts.

\begin{tabular}{|c|c|c|c|}
\hline Fiber & Bile salt & $\begin{array}{c}\mathrm{k}_{1} \\
\left(\mathrm{l} / \mathrm{molx} 10^{4}\right)\end{array}$ & $\begin{array}{c}\mathrm{k}_{2} \\
\left(\mathrm{~mol} / \mathrm{g} \times 10^{-6}\right)\end{array}$ \\
\hline \multirow[t]{3}{*}{ Rikkunshito } & Cholate & 0.14 & 748.0 \\
\hline & Deoxycholate & 0.25 & 752.0 \\
\hline & $\begin{array}{l}\text { Taurodeoxycholate } \\
\text { (Taurocholate) }\end{array}$ & 0.079 & 89.0 \\
\hline Cellulose & Deoxycholate & 0.31 & 5.23 \\
\hline
\end{tabular}


both had maximum binding capacities of $89.0 \times 10^{-6} \mathrm{~mol} / \mathrm{g}$. These results suggest that rikkunshito has high binding capacities for more hydrophobic bile salts compared with hydrophilic bile salts.

Under our HPLC conditions, the hydrophobicity indices were $\mathrm{DCA}>\mathrm{CA}>\mathrm{T}-\mathrm{DCA}$ or T-CA.

\section{Discussion}

Rikkunshito has been shown to promote adaptive gastric relaxation (28) and to facilitate gastric emptying (29). In addition, other pharmacological properties of rikkunshito have been reported, including reducing distal esophageal acid exposure by improving esophageal acid clearance $(30,31)$ and promoting adaptive relaxation $(28,30)$. This herbal drug may also aid the amelioration of GERD symptoms and these effects could be considered to be caused by the prokinetic actions of rikkunshito. One possible mechanism for these therapeutic effects is an increase in the plasma-active ghrelin levels, which stimulate gastric motility (16). However, it is possible that there are other mechanisms whereby rikkunshito improves GERD or DGER symptoms, possibly involving the bile acids.

Rikkunshito is a type of dietary fiber derived from medicinal plants. Early evidence that dietary fiber binds to cytotoxic bile salts was presented by Eastwood and Hamilton (32). It has since been well-documented that fiber is able to adsorb bile salts and thus eliminate bile salts from the digestive tract.

We have studied the role of bile salts in the gut lumen when considering the pathogenesis and disease-promoting factors for experimental and clinical gastrointestinal diseases $(25,26,33-37)$. In the course of these investigations, it has been revealed that bile salts exhibit mainly cytotoxic but also certain stimulatory effects towards the intestinal epithelium (38). In addition, previous clinical studies have suggested that toxic secondary bile acid fractions were detected more frequently in patients with symptoms of GERD. This study also indicated that reflux mixed with gastric acid and bile acid is more harmful than gastric acid reflux alone, with a possible toxic synergism (39). However, the effects of bile salts on the esophageal mucosa are less well understood. Therefore, we investigated whether rikkunshito could adsorb bile salts.

In the present study, we found that rikkunshito swells in distilled water. This phenomenon was accompanied by a high capacity to adsorb bile salts compared with $\alpha$-cellulose ( 150-fold greater; Table I). In general, hydrophobic bile salts bind preferentially to fiber (40). As expected, rikkunshito exhibited high binding capacities for hydrophobic bile salts (DCA) compared with hydrophilic bile salts (T-DCA) ( 8.5-fold greater).

In this study, we compared the binding capacity of rikkunshito with that of cholestyramine. Cholestyramine is an anion-exchange resin and is used clinically to achieve anti-hypercholesterolemia effects. Since cholestyramine has a high binding capacity for bile salts (27), it is able to eliminate bile salts present in the digestive tract. As bile salts are biosynthesized from cholesterol, serum cholesterol levels are eventually reduced. The maximum binding capacities of rikkunshito for DCA reached $20 \%$ of that of cholestyramine. In addition, according to our previous study rikkunshito has
30 times the maximum binding capacity for DCA as the clinically-used adsorbent AST-120 (26).

Taken together, it is possible that rikkunshito is not only involved in gastric emptying through its prokinetic action, but may also reduce the bile acid exposure of the esophageal mucosa by adsorbing bile salts. Therefore, rikkunshito may be an effective drug for the treatment of refractory GERD and DGER. This effect could be beneficial for other diseases, including esophageal cancer, as it has been suggested that duodenal juice, including bile salts, stimulates esophageal stem cells to induce Barrett's esophagus and esophageal adenocarcinomas in experimental models (41).

In conclusion, it has become clear for the first time that rikkunshito exhibits a high adsorbing capacity for bile salts, especially hydrophobic and cytotoxic bile salts including DCA and CA. This adsorbing capacity may contribute in part to the therapeutic efficacy of rikkunshito in the treatment of GERD and DGER patients.

\section{References}

1. Dent J, El-Serag HB, Wallander MA and Johansson S: Epidemiology of gastro-oesophageal reflux disease: a systematic review. Gut 54: 710-717, 2005.

2. Inadomi JM, McIntyre L, Bernard L and Fendrick AM: Step-down from multiple- to single-dose proton pump inhibitors (PPIs): a prospective study of patients with heartburn or acid regurgitation completely relieved with PPIs. Am J Gastroenterol 98: 1940-1944, 2003.

3. Carlsson R, Dent J, Watts R, et al: Gastro-oesophageal reflux disease in primary care: an international study of different treatment strategies with omeprazole. International GORD Study Group. Eur J Gastroenterol Hepatol 10: 119-124, 1998.

4. Crawley JA and Schmitt CM: How satisfied are chronic heartburn sufferers with their prescription medications? Results of the patient unmet needs study. J Clin Outcomes Manag 7: 29-34, 2000.

5. The Gallup Organization. The 2000 Gallup Study of Consumers' Use of Stomach Relief Products. Princeton: Gallup Organization, 2000.

6. Vaezi MF, Lacamera RG and Richter JE: Validation studies of Bilitec 2000: an ambulatory duodenogastric reflux monitoring system. Am J Physiol 267: G1050-1057, 1994.

7. Sifrim D: Acid, weakly acidic and non-acid gastro-oesophageal reflux: differences, prevalence and clinical relevance. Eur J Gastroenterol Hepatol 16: 823-830, 2004.

8. Sifrim D, Castell DO, Dent J, et al: Gastro-oesophageal reflux monitoring: review and consensus report on detection and definitions of acid, non-acid, and gas reflux. Gut 53: 1024-1031, 2004.

9. Netzer P, Gut A, Brundler R, et al: Esophageal visceral sensitivity to bile salts in patients with functional heartburn and in healthy control subjects. Aliment Pharmacol Ther 15: 1375-1384, 2001.

10. Todd JA, Basu KK and de Caestecker JS: Normalization of oesophageal $\mathrm{pH}$ does not guarantee control of duodenogastrooesophageal reflux in Barrett's oesophagus. Aliment Pharmacol Ther 21: 969-975, 2005.

11. Pace F, Sangaletti O, Pallotta S, et al: Biliary reflux and non-acid reflux are two distinct phenomena: a comparison between 24-hour multichannel intraesophageal impedance and bilirubin monitoring. Scand J Gastroenterol 42: 1031-1039, 2007.

12. Tatsuta $\mathrm{M}$ and Iishi $\mathrm{H}$ : Effect of treatment with Liu-Jun-Zi-Tang (TJ-43) on gastric emptying and gastrointestinal symptoms in dyspeptic patients. Aliment Pharmacol Ther 7: 459-462, 1993.

13. Tomono $\mathrm{H}$, Ito $\mathrm{Y}$ and Watanabe T: Successful antiemetic treatment of Tsumura rikkunshi-to extract granules for ethical use in addition to other antiemetic agents in neoadjuvant chemotherapy for an advanced breast cancer patient. Jpn J Cancer Chemother 33: 1129-1131, 2006.

14. Oka T, Tamagawa Y, Tamagawa Y, Hayashida S, Kaneda Y, Kodama N, et al: Rikkunshi-to attenuates adverse gastrointestinal symptoms induced by fluvoxamine. Biopsychosoc Med 15: 1-21, 2007. 
15. Kawahara H, Kubota A, Hasegawa T, Okuyama H, Ueno T, Ida S, et al: Effects of rikkunshito on the clinical symptoms and esophageal acid exposure in children with symptomatic gastroesophageal reflux. Pediatr Surg Int 23: 1001-1005, 2007.

16. Hattori T: Rikkunshito and ghrelin. Int J Pept: pii 283549, Jan 26 , 2010 (Epub ahead of print).

17. Cummings JH, Hill MJ and Jenkins DJA: Changes in fecal composition and colonic function due to cereal fiber. Am J Clin Nutr 29: 1473-1486, 1976

18. Kanauchi O, Hitomi Y and Agata K: Germinated barley foodstuff improves constipation induced by loperamide in rats. Biosci Biotechnol Biochem 62: 1788-1790, 1998.

19. Schneeman BO and Gallaher DD: Dietary fiber. In: Present Knowledge in Nutrition. Ziegler EE and Filer LJ (eds). 7th edition. International Life Science Institute Nutritional Foundation, Washington, pp87-97, 1996.

20. Oakenfull DG and Fenwick DE: Adsorption of bile salts from aqueous solution by plant fiber and cholestyramine. Br J Nutr 40 : 299-309, 1978

21. Araki Y, Kanauchi O, Sugihara H, Fujiyama Y and Hattori T: Germinated barley foodstuff suppresses dextran sulfate experimental colitis in rats: The role of mast cells. Int J Mol Med 19: 257-262, 2007.

22. Kanauchi O, Fujiyama Y, Mitsuyama K, Araki Y, Ishii T, Nakamura T, Hitomi Y, Agata K, Saiki T, Andoh A, Toyonaga A and Bamba T: Increased growth of Bifidobacterium and Eubacterium by germinated barley foodstuff, accompanied by enhanced butyrate production in healthy volunteers. Int J Mol Med 3: 175-179, 1999

23. Kanauchi O, Mitsuyama K, Homma T, Takahama K, Fujiyama Y, Andoh A, Araki Y, Suga T, Hibi T, Naganuma M, et al: Treatment of ulcerative colitis patients by long-term administration of germinated barley foodstuff: multi-center open trial. Int J Mol Med 12: 701-704, 2003.

24. Hanai H, Kanauchi O, Mitsuyama K, Andoh A, Takeuchi K, Takayuki I, Araki Y, Fujiyama Y, Toyonaga A, Sata M, et al: Germinated barley foodstuff prolongs remission in patients with ulcerative colitis. Int J Mol Med 13: 643-647, 2004.

25. Araki Y, Andoh A, Fujiyama Y, Kanauchi O, Takenaka K, Higuchi A and Bamba T: Germinated barley foodstuff exhibits different adsorption properties for hydrophilic versus hydrophobic bile acids. Digestion 64: 248-254, 2001.

26. Araki A, Tsujikawa T, Andoh A, Sasaki M, Fujiyama $Y$ and Bamba T: The therapeutic effects of an oral adsorbent on the acute dextran sulfate sodium-induced colitis and its recovery phase in rats, especially effects of the elimination of bile acids in gut lumen. Dig Liver Dis 32: 691-698, 2000.

27. Johns WH and Bates TR: Quantification of the binding tendencies of cholestyramine. I. Effect of structure and added electrolytes on the binding of unconjugated and conjugated bile-salt anions. J Pharm Sci 58: 179-183, 1969.
28. Hayakawa T, Arakawa T, Kase Y, Akiyama S, Ishige A, Takeda S, et al: Liu-Jun-Zi-Tang, a kampo medicine, promotes adaptive relaxation in isolated guinea pig stomachs. Drugs Exp Clin Res 25: 211-218, 1999.

29. Kido T, Nakai Y, Kase Y, Sakakibara I, Nomura M, Takeda S, et al: Effects of rikkunshi-to, a traditional Japanese medicine, on the delay of gastric emptying induced by $\mathrm{N}(\mathrm{G})$-nitro-L-arginine. J Pharmacol Sci 98: 161-167, 2005.

30. Suzuki H, Inadomi JM and Hibi T: Japanese herbal medicine in functional gastrointestinal disorders. Neurogastroenterol Motil 21: 688-696, 2009.

31. Kawahara H, Mitani Y, Nomura M, et al: Impact of rikkunshito, an herbal medicine, on delayed gastric emptying in profoundly handicapped patients. Pediatr Surg Int 25: 987-990, 2009.

32. Eastwood M and Hamilton D: Studies on the adsorption of bile salts to non-absorbed components of the diet. Biochim Biophys Acta 152: 165-173, 1968.

33. Araki Y, Andoh A, Tsujikawa T, Fujiyama Y and Bamba T: Alterations in intestinal microflora, faecal bile acids and short chain fatty acids in dextran sulphate sodium-induced experimental acute colitis in rats. Eur J Gastroenterol Hepatol 3: 107-112, 2001.

34. Araki Y, Fujiyama Y, Andoh A, Nakamura F, Shimada M, Takaya $\mathrm{H}$ and Bamba T: Hydrophilic and hydrophobic bile acids exhibit different cytotoxicities through cytolysis, interleukin-8 synthesis and apoptosis in the intestinal epithelial cell lines. IEC-6 and Caco-2 cells. Scand J Gastroenterol 36: 533-539, 2001.

35. Araki Y, Andoh A, Bamba H, Yoshikawa K, Doi H, Komai Y, Higuchi A and Fujiyama Y: The cytotoxicity of hydrophobic bile acids is ameliorated by more hydrophilic bile acids in intestinal cell lines IEC-6 and Caco-2. Oncol Rep 10: 1931-1936, 2003.

36. Araki Y, Katoh T, Ogawa A, Bamba S, Andoh A, Koyama S, Fujiyama Y and Bamba T: Bile acid modulates transepithelial permeability via the generation of reactive oxygen species in the Caco-2 cell line. Free Radic Biol Med 39: 769-780, 2005.

37. Araki Y, Mukaisyo KI, Sugihara H, Fujiyama Y and Hattori T: Detection of N-nitroso-bile acids at $285 \mathrm{~nm}$ in reverse-phase HPLC. J Sep Sci 31: 2827-2830, 2008.

38. Araki Y, Andoh A, Sasaki A, Shimada M, Bamba S, Fujino S and Fujiyama Y: Dietary bile acids inhibit potentially elemental diet-induced small intestinal atrophy in rats. Int J Mol Med 10: 623-626, 2002

39. Nehra D, Howell P, Williams CP, Pye JK and Beynon J: Toxic bile acids in gastro-oesophageal reflux disease: influence of gastric acidity. Gut 45: 598-602, 1999

40. Kern F, Birkner HJ and Ostrower VS: Binding of bile acids by dietary fiber. Am J Clin Nutr 31: S175-179, 1978.

41. Miyashita T, Ohta T, Fujimura T, Ninomiya I, Fushida S, Hattori T and Miwa K: Duodenal juice stimulates oesophageal stem cells to induce Barrett's oesophagus and oesophageal adenocarcinoma in rats. Oncol Rep 15: 1469-1475, 2006. 